\title{
Dual-band double-negative-index fishnet metamaterial at millimeter-waves
}

\author{
Miguel Navarro-Cía, ${ }^{1,2, *}$ Carlos García-Meca, ${ }^{3}$ Miguel Beruete, ${ }^{2}$ Alejandro Martínez, ${ }^{3}$ and Mario Sorolla ${ }^{2}$ \\ ${ }^{1}$ Experimental Solid State Group, Imperial College London, London SW7 2AZ, UK \\ ${ }^{2}$ Millimeter and Terahertz Waves Laboratory, Universidad Pública de Navarra, 31006 Pamplona, Spain \\ ${ }^{3}$ Nanophotonics Technology Center, Universitat Politècnica de València, 46022 Valencia, Spain \\ *Corresponding author: m.navarro@imperial.ac.uk
}

Received May 10, 2011; revised September 20, 2011; accepted September 29, 2011;

posted September 30, 2011 (Doc. ID 147273); published October 28, 2011

\begin{abstract}
An effective negative refractive index (NRI) is demonstrated and experimentally verified for the first two propagation bands of a fishnet-like metamaterial at millimeter-wave frequencies. The dual-band NRI behavior is achieved by engineering the diffraction order $( \pm 1, \pm 1)$ associated with the internal mode supported between holey layers to correspond with the second propagation band. In addition to the experimental interferometric technique that accounts for the handedness of the propagation, numerical results are given to predict the dual-band effective NRI and to confirm dual-band negative refraction for a prism composed of the proposed metamaterial. (c) 2011 Optical Society of America

OCIS codes: $\quad 050.2065,160.1245,160.3918,240.6690,290.3030$.
\end{abstract}

Metamaterials (mtms) [1] hold promise for both basic science and technology due to their linked new physics such as negative refractive index (NRI) [2] or perfect lensing [3]. Within the past years, an unexpected theoretical and experimental development has helped to strengthen the evidence that the effective NRI observed in fishnet mtms (stacked hole arrays) $[\underline{4}, \underline{5}]$ has a strong connection with extraordinary transmission phenomenon [5-7]. Fishnet mtms, first reported at infrared frequencies $[\underline{4}, \overline{8}]$, have been widely studied in parallel at microwaves-toterahertz where they have been termed extraordinary transmission mtms [5,, 9$]$ (because of the aforementioned connection) and infrared-to-visible frequencies [10-13] because they substantially outperform other mtms. Most of the existing studies have ultimately confirmed that the general underlying physics relies primarily on diffraction orders of complex surface waves [14-19] that can be either external or internal (Floquet-Bloch guided mode of the waveguide formed by pairs of holey metals) complex surface waves $[11,12,15,18-20]$. Indeed, this interpretation allowed some authors of this Letter to engineer the $( \pm 1, \pm 1)$ diffraction order of the internal mode to become the second propagation band of the fishnet $\mathrm{mtm}[\underline{11}, \underline{12}, \underline{18}]$, achieving effective NRI for the first two propagation bands [the first one associated to the $(0, \pm 1]$ diffraction order of the internal mode). Notice that in [19], no dual-band double-negative-index behavior is displayed under normal incidence for the first two propagation modes.

Recently, several authors of this Letter reported an experimental verification of the above-mentioned fishnet resonance $( \pm 1, \pm 1)$ at visible wavelengths [18]. In this spectral range, only amplitude measurements are usually carried out due to the difficulty of capturing the phase response. Here, we demonstrate experimentally at millimeter-waves the backward-wave nature of the aforementioned lowest propagation bands from the phase response of the fishnet structure as a function of the number of unit cells in the propagation direction. Moreover, this work reinforces the view that the phenomenon is not limited to a particular class of complex surface wave such as surface plasmon polaritons, but to the whole framework of complex surface waves.

The sketch of the fishnet mtm under study can be seen in Fig. 1, together with the fabricated prototype. The unit cell parameters are: in-plane periodicities $d_{x}=d_{y}=$ $d=2.5 \mathrm{~mm}$, stack periodicity $d_{z}=0.525 \mathrm{~mm}\left(\approx 0.13 \lambda_{0}\right.$ and $0.19 \lambda_{0}$ for the first and second propagation band, respectively), hole diameter $a=1.1 \mathrm{~mm}$, metal thickness $t=0.035 \mathrm{~mm}$, and dielectric permittivity of the dielectric spacing $\varepsilon_{r}=2.43-j 0.022$. We define a single functional layer (FL) as metal-dielectric-metal, whereas successive functional layers consist of $2 N+1$ alternating metal and dielectric stacked hole arrays with $N=2$, 3, etc. (for instance, two FLs comprise metal-dielectric-metaldielectric-metal) [11].

The dependence of the retrieved effective index of refraction $n_{z}$ [21] as a function of the number of FLs is displayed in Fig. 2(a). It should be noted that physically meaningful material parameters are linear response functions that must be independent of the applied electric field and independent of the geometrical size and shape of a medium sample [22]. Therefore, the effective index of refraction assigned to this finite (in number of metal layers) mesoscopic stack by this procedure should be interpreted with some precautions [23]. For comparison purposes, we calculated the dispersion diagram of the
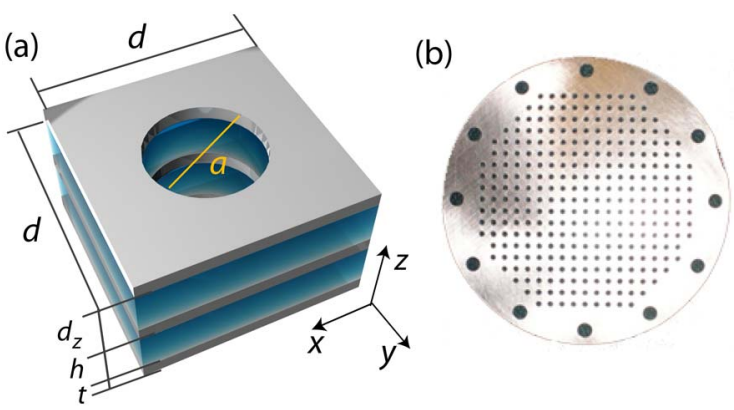

Fig. 1. (Color online) (a) Geometrical dimensions of the considered fishnet structure (picture of two unit cells). (b) Fabricated prototype. 


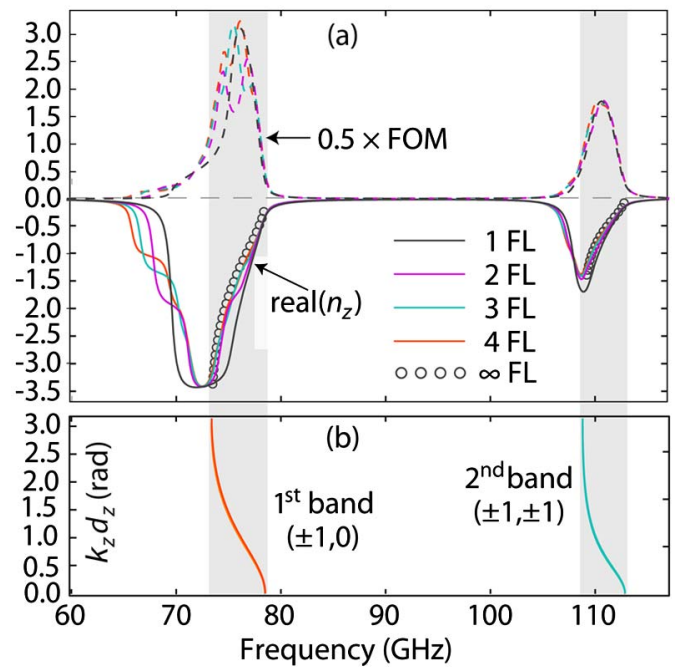

Fig. 2. (Color online) (a) Retrieved effective refractive index, for different number of FLs. Solid and dashed lines represent the real part of $n_{z}$ and the figure of merit, $\mathrm{FOM}=$ $\left|\operatorname{Re}\left(n_{z}\right) / \operatorname{Im}\left(n_{z}\right)\right|$, respectively. The circles correspond to the index derived from the dispersion diagram. (b) Dispersion diagram.

ideal infinite structure through an Eigen-mode analysis. The first two propagation bands are plotted in Fig. 2(b), where we can observe their backward nature. The effective $n_{z}$ derived from the dispersion diagram $\left(n_{z}=\operatorname{sgn}\left(\partial \omega / \partial k_{z}\right) k_{z} c_{0} / \omega\right.$, where $\omega=2 \pi f$ is angular frequency and $c_{0}$ the speed of light in vacuum) is also plotted in Fig. 2(a), which is in excellent agreement with the retrieved $\overline{n_{z}}$ corresponding to the finite structure. Note that losses are not taken into account in the Eigenmode analysis. As a consequence, the low-frequency part of each band in Fig. 2(a) is a forbidden region in the dispersion diagram, since at these frequencies propagation is dominated by losses.

The internal modes responsible for these two bands can be clearly identified by inspection of the electric field $E$ distribution at the corresponding frequencies. In Fig. 3(a) we depict the $E$-field intensity in a $x y$ plane halfway between two metal plates for the one FL case. The field patterns are unequivocally those of the first and second diffraction orders of the internal mode. Moreover, the plots of $E$ in the $y z$ plane crossing the center of the holes confirm that the fields are concentrated between metals at these frequencies.

To provide an even more solid verification, the effective negative refractive behavior was examined experimentally. The stack shown in Fig. 1(b) was fabricated using ARLON CUCLAD $250 \mathrm{LX}$ and a laser-based printed circuit board production. For the experimental characterization, an $\mathrm{AB}-$ Millimetre ${ }^{\mathrm{TM}}$ vector network analyzer and a quasi-optical bench were used.

Figures 3(b)-3(d) show both the complex experimental data's magnitude and phase response. In agreement with the numerical calculations, two prominent bands come up within the $W$-band of the millimeter-waves. The first one associated to the $(0, \pm 1)$ internal mode, whereas the second linked to the $( \pm 1, \pm 1)$ internal mode. The band spectral positions of the measurements coincide with the numerical calculations, except for a slight
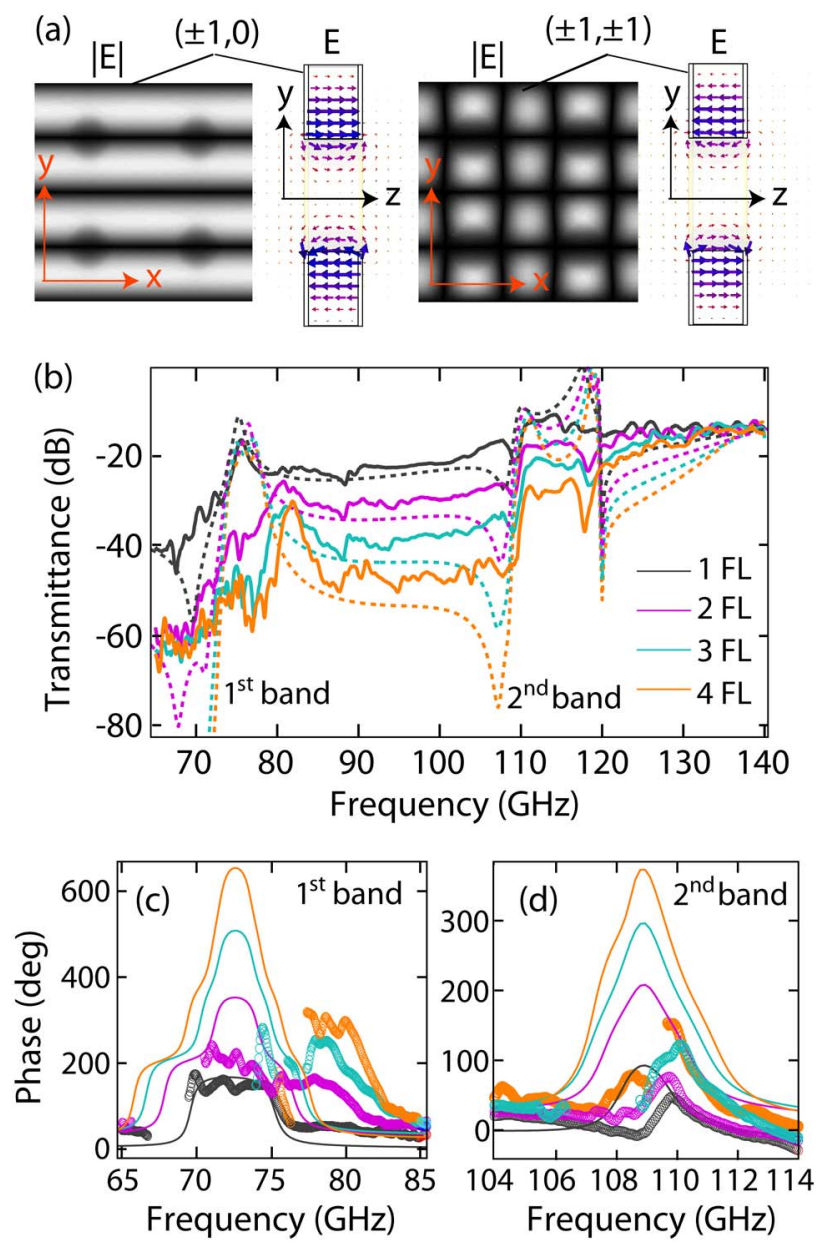

Fig. 3. (Color online) (a) Electric field at the center frequency of both bands at two different two-dimensional cuts. The incident wave is polarized with the $E$-field along the $y$-axis. (b) Measured (solid lines) and simulated (dashed lines) transmission spectra. (c) First and (d) second band measured (circles) and simulated (solid lines) phase response on transmission.

frequency increase of the first band, which can be safely ascribed to nonperfect assembly of the FLs. The growth of the stack is performed by screwing (at the framework) additional metal-dielectric slabs or FLs which likely leaves small air gaps between stacked layers. This may cause a slight reduction of the effective dielectric permittivity of the interspacing. It is noteworthy that losses experienced by the second band are lower than the first band. Quantitatively, it can be argued that the holes are more subwavelength for the first band and thus narrower peaks are expected, which are more susceptible to losses. Alternatively, the Drude response of the permittivity together with the double Lorentz model for the permeability can explain the insertion losses from an impedance mismatch perspective. Assuming that both Lorentz responses have identical strength, the effective electric permittivity may not reach negative values low enough to be similar to the effective magnetic permeability. However, at the second resonance it reaches lower values that may balance the effective magnetic permeability, achieving thus impedance matching. Indeed, according to the retrieval method, the maximum wave impedance of four FLs is 0.075 and 0.17 for the first 


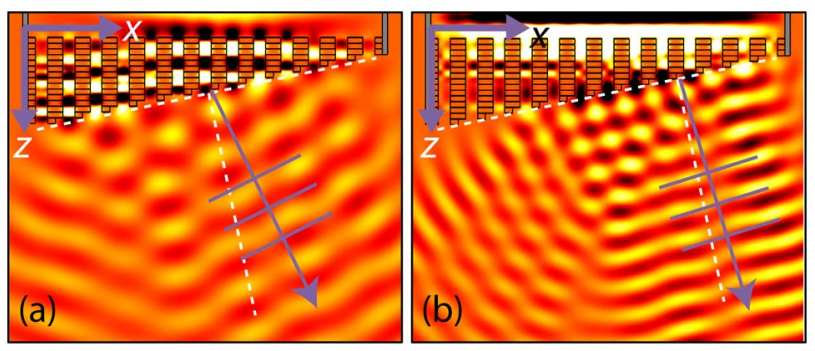

Fig. 4. (Color online) Refraction in a fishnet $\mathrm{mtm}$ prism for (a) the first and (b) the second band. The interface and its normal are plotted in dashed line and the power flow direction with a purple arrow.

and second band, respectively. Therefore, the Fresnel reflection losses are higher at the first band, justifying the higher measured insertion losses in this band.

In order to assign a backward- or forward-wave propagation direction from the experimental data one should inspect the phase response [see Fig. 3(c) and 3(d)]. For backward-wave/forward-wave propagation, the phase response increases/decreases with the number of FLs because of the $\exp \left(-j k_{z} \Delta l\right)$ dependence of the transmission, where $\Delta l$ is the length of the stack and $k_{z}$ is the wave-number in the stack. In the case of effective NRI $\operatorname{mtms} k_{z}<0$ and thus the dependence is $\exp \left(+j\left|k_{z}\right| \Delta l\right)$. With this description in mind, the response of the phase shown in Figs. 3(c) and 3(d) is clearly in accordance with backward-waves propagation, that is, effective negative $n_{z}$. The simulated phase response in transmission is also included in Figs. 3(c) and 3(d) and exhibits the same tendency as the measured results. Note that the phase response for very low values of transmittance, where the measurement of phase is challenging, has been removed from the plot to avoid misinterpretation.

As a final check, we simulate a prism based on the fishnet $\mathrm{mtm}$. To simplify the numerical effort, the simulations deal with a two-dimensional prism. This allows us to confirm straightforwardly the negative refraction associated with the first two propagation bands. From the numerical results shown in Fig. 4 , it can be seen that the proposed fishnet $\mathrm{mtm}$ supports one-dimensional effective negative refraction in both bands since the outgoing beam deflects towards the normal.

In summary, we have demonstrated experimentally at millimeter-wave frequencies the unusual phase response associated with an effective NRI for the two first propagation bands of a fishnet mtms. This effect is achieved by tuning the parameters in such a way that the $( \pm 1, \pm 1)$ diffraction order of the internal mode is the second propagation band. The index of refraction retrieved from the scattering parameters of numerical simulations shows good agreement with the index derived from the structure eigenmodes and both are confirmed by experimental measurements. Furthermore, negative refraction in both bands is numerically demonstrated with a prism. This experimental confirmation is another stepping stone in the path to enrich the properties of mtms, making them attractive for practical applications at frequencies such as millimeter-wave or terahertz.
Authors are grateful to R. Ortuño and F.J. RodríguezFortuño (Valencia Nanophotonics Technology Center) for their helpful comments, to G. Crespo (Universidad Pública de Navarra) for sample fabrication, and to S. Hanham and A. Demetriadou (Imperial College London) for improving the readability of this Letter. Work supported by Spanish Government and European Union (EU) funds under contracts Consolider "Engineering Metamaterials" CSD2008-00066, TEC2008-06871-C02, and Valencian Government (contract PROMETEO-2010087). M. N.-C. acknowledges financial support from Leverhulme Trust. C. G.-M. acknowledges financial support from grant Formación del Profesorado Universitario (FPU) of Ministerio de Ciencia e Innovación (MICINN).

\section{References}

1. L. Solymar and E. Shamonina, Waves in Metamaterials (Oxford University, 2009).

2. V. G. Veselago, Sov. Phys. Usp. 10, 509 (1968).

3. J. B. Pendry, Phys. Rev. Lett. 85, 3966 (2000).

4. S. Zhang, W. Fan, N. C. Panoiu, K. J. Malloy, R. M. Osgood, and S. R. J. Brueck, Phys. Rev. Lett. 95, 137404 (2005).

5. M. Beruete, M. Sorolla, and I. Campillo, Opt. Express 14, 5445 (2006).

6. T. W. Ebbesen, H. J. Lezec, H. Ghaemi, T. Thio, and P. A. Wolf, Nature 391, 667 (1998).

7. M. Beruete, M. Sorolla, I. Campillo, J. S. Dolado, L. Martín-Moreno, J. Bravo-Abad, and F. J. García-Vidal, Opt. Lett. 29, 2500 (2004).

8. G. Dolling, C. Enkrich, M. Wegener, C. M. Soukoulis, and S. Linden, Science 312, 892 (2006).

9. M. Navarro-Cía, M. Beruete, M. Sorolla, and I. Campillo, Opt. Express 16, 560 (2008).

10. J. Valentine, S. Zhang, T. Zentgraf, E. Ulin-Avila, D. A Genov, G. Bartal, and X. Zhang, Nature 455, 376 (2008).

11. C. García-Meca, R. Ortuño, F. J. Rodríguez-Fortuño, J. Martí, and A. Martínez, Opt. Lett. 34, 1603 (2009).

12. R. Ortuño, C. García-Meca, F. J. Rodríguez-Fortuño, J. Martí, and A. Martínez, Phys. Rev. B 79, 075425 (2009).

13. S. Xiao, U. K. Chettiar, A. V. Kildishev, V. P. Drachev, V. M. Shalaev, Opt. Lett. 34, 3478 (2009).

14. A. Ishimaru, Electromagnetic Wave Propagation, Radiation, and Scattering (Prentice Hall, 1991).

15. A. Mary, S. G. Rodrigo, F. J. García-Vidal, L. Martin-Moreno, Phys. Rev. Lett. 101, 103902 (2008).

16. M. Beruete, M. Navarro-Cía, M. Sorolla, and I. Campillo, Phys. Rev. B 79, 195107 (2009).

17. M. Beruete, M. Navarro-Cía, M. Sorolla, and I. Campillo, New J. Phys. 12, 063037 (2010).

18. C. García-Meca, J. Hurtado, J. Martí, A. Martínez, W. Dickson, and A. V. Zayats, Phys. Rev. Lett. 106, 067402 (2011).

19. M. Beruete, M. Navarro-Cía, and M. Sorolla, "High numerical aperture and low-loss negative refraction based on the fishnet rich anisotropy," Photonic. Nanostruct: Fundam. Appl. (to be published).

20. J. Yang, C. Sauvan, H. T. Liu, and P. Lalanne, Phys. Rev. Lett. 107, 043903 (2011).

21. X. Chen, T. M. Grzegorczyk, B. Wu, J. Pacheco Jr., and J. A. Kong, Phys. Rev. E 70, 016608 (2004).

22. L. Landau and E. M. Lifschitz, Electrodynamics of Continuous Media (Elsevier, 1984).

23. C. Menzel, T. Paul, C. Rockstuhl, T. Pertsch, S. Tretyakov, and F. Lederer, Phys. Rev. B 81, 035320 (2010). 
